\title{
Motivational interviewing for modifying diabetes risk: a randomised controlled trial
}

\author{
Colin J Greaves, Andrew Middlebrooke, Lucy O'Loughlin, Sandra Holland, Jane Piper, \\ Anna Steele, Tracy Gale, Fenella Hammerton and Mark Daly
}

\begin{abstract}
Background

Around $10-15 \%$ of adults aged over 40 years have pre-diabetes, which carries a high risk of progression to type 2 diabetes. Intensive lifestyle intervention reduces progression by as much as $58 \%$. However, the cost and personnel requirements of these interventions are major obstacles to delivery in NHS primary care.

Aim

To assess the effectiveness of a low-cost intervention, delivered in primary care by non-NHS staff, to reduce the risk of diabetes through weight loss and physical activity.

\section{Design of study}

Pragmatic single-blind randomised controlled trial with researchers and statistician blinded to group allocation.

Setting

UK primary care.

\section{Method}

One-hundred and forty-one participants with a body mass index of $28 \mathrm{~kg} / \mathrm{m}^{2}$ or more, but without diabetes or heart disease, received either information leaflets or individual behavioural counselling using motivational interviewing techniques. The intervention was delivered by five counsellors recruited from the local community. The primary outcomes were the proportions of participants meeting predefined targets for weight loss (5\%) and moderate physical activity (150 minutes/ week) after 6 months.

\section{Results}

Using intention-to-treat analysis, more people in the intervention group achieved the weight-loss target (24\% versus $7 \%$ for controls; odds ratio $[\mathrm{OR}]=3.96$; $95 \%$ confidence interval $[\mathrm{Cl}]=1.4$ to 11.4 ; number needed to treat $[N N T]=6.1(95 \% \mathrm{Cl}=4$ to 21$)$. The proportion achieving the physical activity target did not increase significantly ( $38 \%$ versus $28 \%$ for controls; $\mathrm{OR}=1.6 ; 95 \% \mathrm{Cl}=0.7$ to 3.8 ).

\section{Conclusion}

Short-term weight loss, at a level which, if sustained, is clinically meaningful for reducing diabetes risk, is achievable in primary care, without excessive use of NHS monetary or personnel resources.

\section{Keywords}

behaviour therapy; diabetes mellitus; intervention studies; primary prevention; randomised controlled trial; weight loss.
\end{abstract}

\section{INTRODUCTION}

Recent large studies in the US, China, and Finland have shown that modest changes in physical activity and diet can have a large health impact in terms of preventing progression to type 2 diabetes. ${ }^{1-4}$ Around $15 \%$ of adults aged over 40 years have either impaired fasting glycaemia or impaired glucose tolerance (collectively known as pre-diabetes), ${ }^{5}$ and large numbers may be detected in UK primary care over the next few years, as initiatives for primary prevention of heart disease and (possibly) targeted diabetes screening are implemented.

The problem with the diabetes-prevention studies conducted to date is that to achieve the required behavioural changes has required very intensive interventions. The Finnish Diabetes Prevention Study involved seven individual sessions with a nutritionist, quarterly review sessions, plus separate individual physical activity coaching and behavioural counselling sessions. ${ }^{3}$ The US programme involved 16 weekly individual sessions, followed by an individualised maintenance programme including further individual and group sessions. The intervention was delivered by

CJ Greaves, PhD, senior research fellow, Peninsula Medical School, Exeter. A Middlebrooke, PhD, lecturer, University of Exeter School of Sports and Health Sciences, Exeter. L O'Loughlin, MSc, head of health improvement;

F Hammerton, BSc, cardiac specialist nurse; S Holland, DipMS, health improvement specialist, Devon Primary Care Trust, Exeter. J Piper, RGN, diabetes research nurse; A Steele, MSc, NIHR research training fellow; T Gale, PhD, specialist dietician, Royal Devon and Exeter NHS Foundation Trust, Diabetes and Vascular Medicine, Exeter. M Daly, MD, MRCP, consultant diabetologist, Peninsula Medical School and Royal Devon and Exeter NHS Foundation Trust, Exeter.

Address for correspondence Dr Colin Greaves, Peninsula Medical School (Primary Care), Room 105 Smeall Building, St Luke's Campus, Magdalen Road, Exeter. EX1 2LU. E-mail: Colin.Greaves@pms.ac.uk

Submitted: 21 November 2007; Editor's response: 29 January 2008; final acceptance: 24 April 2008.

() British Journal of General Practice.

This is the full-length article of an abridged version published in print. Cite this article as: Br J Gen Pract 2008; DOI: 10.3399/bjgp08X319648 
registered dieticians, exercise physiologists, professional behavioural psychologists, and health educators. ${ }^{1}$ This intensity of professional-led intervention is not likely to be affordable in UK primary care, and the resources in terms of available staff time are also a major constraint. The need for evaluation of a range of more practical community-based options has been highlighted by key authors in this field. ${ }^{4,6,7}$

One promising approach, which offers a well-defined and evidence-based model for behaviour change, is motivational interviewing. ${ }^{8-11}$ Motivational interviewing focuses on exploring and making explicit the reasons someone might have to engage in a particular behaviour or course of action. Strongly patient-centred interviewing techniques are used to contrast the pros and cons of change, reduce sources of resistance, and identify possible barriers to change. Action plans are then made, which specifically 'situate' the planned change in the context of the person's day-to-day life. The principles and techniques are easy to learn, and well-established training courses are available through a national network of accredited trainers. ${ }^{12}$ The techniques can also be used successfully by nonhealth professionals. ${ }^{13}$

The aim of this study therefore was to see whether changes in weight and physical activity similar to those obtained in the Finnish and US diabetes-prevention studies could be achieved with a simplified intervention, based on motivational interviewing, and delivered by trained health-promotion workers not currently working for the NHS.

\section{METHOD}

\section{Design}

A single-blind, pragmatic, randomised controlled trial was conducted in a community setting, with participants allocated to receive either intervention or 'information only' in addition to any usual care being received. 'Usual care' means that the care of participants was not interfered with in any way outside of the study setting, although changes in medication or new diagnosis of illness during the course of the study were monitored.

\section{Intervention}

Five 'health-promotion counsellors' were trained to deliver motivational interviewing in one-to-one individual consultations. They worked part-time and were selected to have prior experience in promoting physical activity or healthy diet with adults, and not to be current NHS employees. Two were ex-NHS staff (health visitor, rehabilitation nurse) and three were postgraduate students in sports and health science. The training included 2 days on the assessment of existing diet and physical activity, and setting appropriate, individually tailored, diet and activity

\section{How this fits in}

Modest changes in physical activity and diet can have a large health impact in terms of preventing progression to type 2 diabetes. Intensive behavioural interventions have been shown to be effective in achieving and sustaining the required changes. This study shows that short-term weight and physical activity changes can be achieved with a motivational interviewing intervention, delivered by non-health professionals, with relatively low usage of primary care resources. Further research is needed to establish the most practical and cost-effective methods for achieving long-term diabetes prevention in primary care settings.

targets. Dietary recommendations were based on reducing overall calorific intake and portion size, reducing overall fat intake, reducing saturated fat content, and increasing fibre intake. This included aiming to increase ingestion of wholegrain products, vegetables, fruit, low-fat milk, low-fat meat, soft margarines, and vegetable oils rich in monounsaturated fat. Physical activity recommendations focused on increasing overall physical activity within the context of the individual's existing life, rather than specifying a predetermined activity regime. Training in supporting behaviour change consisted of a 2-day course in motivational interviewing, provided by an accredited motivational interviewing trainer. ${ }^{12}$

Participants in the intervention group received up to 11 individual sessions over a 6 month period. A mixture of one-to-one contacts (median 8) and telephone contacts (median 1.5) was received with a mean 34 minutes per contact. Action plans were made and checked/modified at subsequent contacts using relapse-management/relapse-prevention techniques, and targets were increased gradually to build/reinforce confidence over time. Participants were also encouraged to self-monitor their own weight, activity, and energy levels. A specific aim of the intervention was to encourage participants to develop sustainable cognitive and behavioural skills for managing their diet and physical activity. The fixed-term nature of the intervention was emphasised, and the length of appointments tapered off to allow a planned withdrawal of support and to minimise dependence on the therapist. Interventions were delivered in a consulting room at a local community hospital in Devon. Intervention fidelity was checked by analysing transcripts of interviews conducted by the counsellors with an actor who responded based on a given scenario. One behavioural scientist and one nurse consultant with experience of using motivational interviewing techniques marked the transcripts, using the Behaviour Change Counselling Index (BECCl). ${ }^{14}$ The standard achieved was deemed (in the opinion of the assessors) to be satisfactory for all counsellors. Attendance records indicated that $96 \%$ of the 
intervention group received at least three sessions of treatment, and $79 \%$ received six or more sessions. Reviews of motivational interviewing suggest that at least two sessions and at least 60 minutes of contact time are required for benefit. ${ }^{15,16}$ Hence, the majority of intervention participants received a therapeutic dose of treatment.

\section{Control}

Control group participants received a standardised information pack promoting similar diet and physical activity recommendations to those above. Specifically, British Heart Foundation health-promotion materials were used; the NHS Smoking Cessation Service 'Green Book'; and locally produced information on physical activity and local 'walk and talk' activities. At the end of the study (post data collection), controls received two individual sessions with the study counsellors.

\section{Participants}

The study recruited adults (age $\geq 18$ years) identified by their GP as having a body mass index (BMI) of $28 \mathrm{~kg} / \mathrm{m}^{2}$ or more (a level clearly associated with increased diabetes risk $)^{17}$ recorded in the last 10 years. Participants were excluded if they had existing diabetes, heart disease, or severe joint problems, if they felt they would be unable to engage in at least moderate physical activity, if the GP felt the patient to be unsuitable (for example, severe uncontrolled hypertension, dementia, or mental illness), or if they did not speak English fluently.

\section{Measures}

Outcomes were measured at baseline and 6 months after the first consultation. Baseline demographic data on age, sex, and level of education (primary, secondary, or degree level) were collected. Participants were also asked about the onset of new medical conditions, medication use, and attendance at other weight-loss or physical activity programmes during the course of the study. The primary outcomes were the proportion of patients achieving the prespecified target of $5 \%$ reduction in weight, and the proportion of patients achieving the UK government target of 150 minutes of moderate activity per week. ${ }^{18}$ Secondary outcomes were weight $(\mathrm{kg})$ and waist circumference $(\mathrm{cm})$, measured using appropriate guidelines. ${ }^{19}$ Physical activity levels were self-reported using the Modifiable Activity Questionnaire (MAQ). ${ }^{20}$

\section{Analysis}

The primary analysis was an intention-to-treat analysis of the proportions achieving the specified targets, using logistic regression with group allocation and baseline values entered into the regression model (except for weight loss, where the baseline value is integral to the measure). Where follow-up data were missing, the baseline value was carried forward (that is, no change from baseline was assumed). Secondary outcomes were compared using analysis of covariance (ANCOVA; with baseline-dependent variable scores entered into the model). Further analyses assessed the sensitivity of both primary and secondary analyses to differences in baseline characteristics (age, sex, and education level) and to other factors that might affect weight (incidence of new illness, or medication that might affect weight, and use of other weight-loss programmes). Analyses were conducted using SPSS (version 14).

\section{Sample size calculation}

The sample size was calculated based on the analysis of primary outcomes (target weight and activity). Estimates of the likely proportions were taken from previous studies (43\% achieving target weight loss and $36 \%$ physical activity in the intervention group versus $13 \%$ and $16 \%$ in the control group, using intention-totreat basis). ${ }^{1,3}$ To have an $80 \%$ chance of detecting these differences, with a (two-sided) 0.05 significance level, 34 patients per group were required for weight, and 73 per group for physical activity.

\section{Procedure}

Participants were recruited between February and June 2006 from two GP surgeries in a semi-rural town in the UK. A list of eligible patients was identified by searching practice databases for people meeting the BMI, age, diabetes, and heart disease criteria. The patients' GPs checked for additional exclusion criteria. Participants were invited by letter to attend group recruitment meetings at a local community hall, and the researchers attempted to confirm attendance by telephone contact. At the meetings, the study was explained in a brief presentation. Refreshments were served and participants could leave at this stage if desired. Written consent was taken individually, using well-spaced desks at one end of the hall. Participants completed baseline questionnaires and then their weight and waist were measured in private rooms.

\section{Randomisation}

Patients were randomly allocated to treatment groups using sealed envelopes, each containing either a control or an intervention slip. These were drawn from a box by a researcher, following completion of baseline measures. A batch of envelopes was prepared at each recruitment session by the chief investigator or the delegated representative, to match the number of participants attending.

\section{Blinding}


Researchers taking weight and waist measures were blind to group allocation (at baseline, measures were taken prior to randomisation, and at follow-up, patients went into a private room for measurement, which involved only a brief interaction with the researcher). Participants were, by necessity, unblinded. The statistician was blinded to group allocation by withholding the key to group labelling until after analysis.

\section{RESULTS}

Of the 247 eligible patients that could be contacted, 141 (57\%) agreed to take part. At the 6-month followup point, 115 patients (82\%) provided weight and waist circumference data, although only 88 (62\%) provided physical activity questionnaires. The recruited sample was broadly similar to the eligible population (mean age 51.9 years, $58 \%$ female), being older by 2.7 years and with a slightly higher proportion of females (64\%). The flow of participants through the study is shown in Figure 1. Table 1 shows the sample characteristics at baseline.

Table 2 shows the outcomes at 6 months. A significantly higher proportion in the intervention group achieved $5 \%$ weight loss (24\% versus $7 \%$ for controls; odds ratio $[\mathrm{OR}]=4.0 ; 95 \%$ confidence interval $[\mathrm{Cl}]=$ 1.4 to 11.4 ). The number needed to treat (NNT) to achieve $5 \%$ weight loss was $6.1(95 \% \mathrm{Cl}=3.6$ to 20.8$)$. A higher proportion in the intervention group achieved the 150-minute physical activity target, although this difference was not significant (38\% versus $28 \%$ for controls; $\mathrm{OR}=1.6 ; 95 \% \mathrm{Cl}=0.7$ to 3.8 ).

Secondary analyses showed a significant difference in weight (adjusted mean difference $1.3 \mathrm{~kg} ; 95 \% \mathrm{Cl}=$ 0.2 to 2.4 ), and a small, non-significant difference in waist circumference between the two groups (adjusted mean difference: $1.6 \mathrm{~cm} ; 95 \% \mathrm{Cl}=-0.01$ to $3.3, P=$ 0.051).

The results showed very little sensitivity to baseline demographic characteristics. A sensitivity analysis excluding six people who developed potentially confounding medical conditions during the study (two thyroid problems, one pre-diabetes with metformin treatment, one heart condition, two musculoskeletal problems), and eight who received alternative weightloss interventions (one orlistat, seven slimming or gymbased programmes) slightly increased the odds ratio for achieving weight loss $(\mathrm{OR}=4.5(95 \% \mathrm{Cl}=1.4$ to 14.6). For those completing the study (per-protocol analysis), 29\% in the intervention group achieved the weight-loss target (versus $9 \%$ in the control group), and $46 \%$ achieved the physical activity target (versus $34 \%$ of controls).

The costs for delivering the intervention were estimated to be $£ 263$ per patient, based on financial records of the direct costs associated with the project

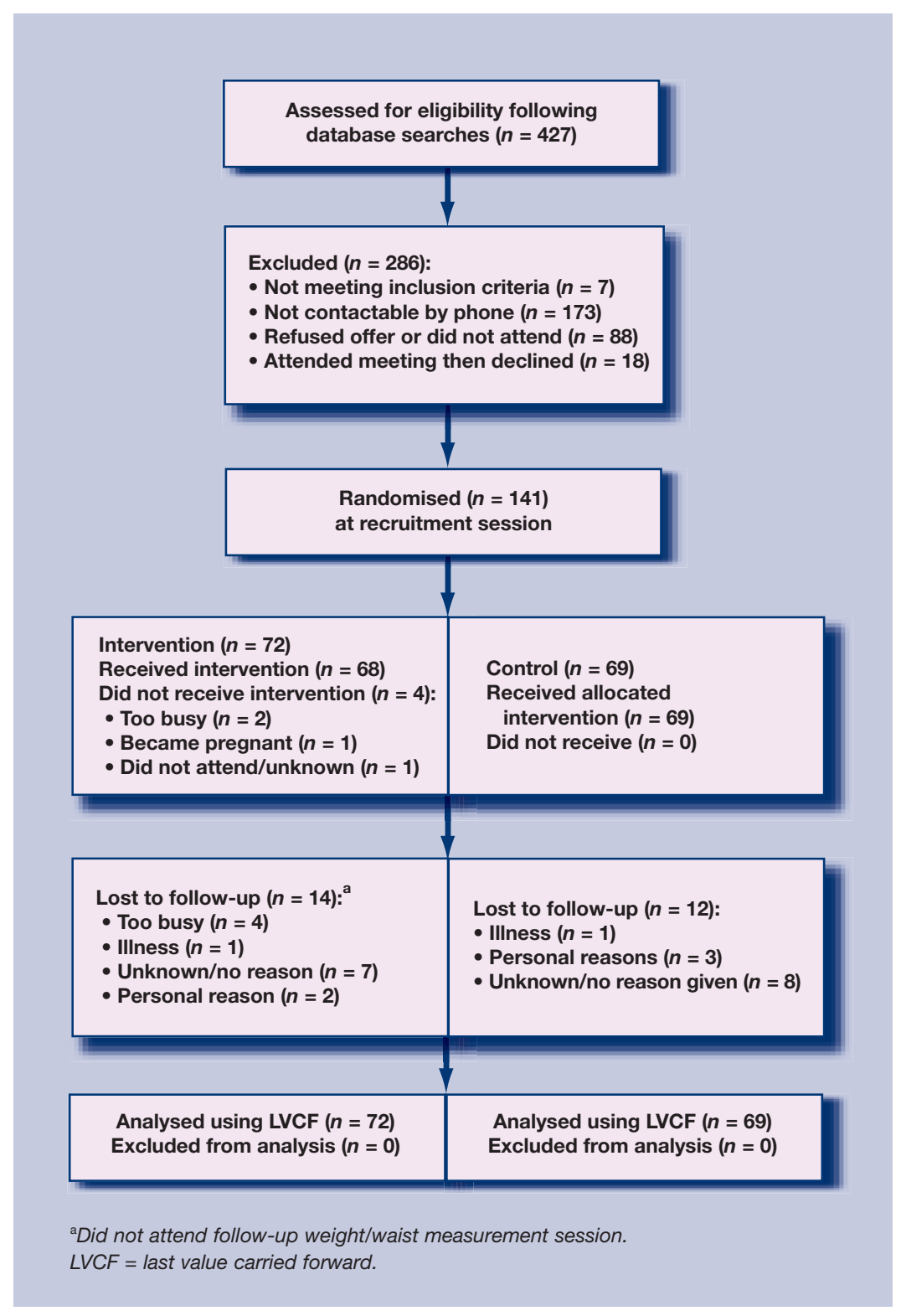

(salaries, supervision/management time, stationery costs) and estimates of indirect costs (consulting room, reception support and hospital overheads, telephone, secretarial support, and general practice costs for the identification and referral of patients) but not project set-up costs (for example, recruitment). Appendix 1

Table 1. Baseline characteristics.

\begin{tabular}{lcc} 
& Intervention $(n=72)$ & Control $(n=69)$ \\
\hline Mean (SD) age, years & $53.3(12.3)$ & $54.5(11.5)$ \\
\hline Female sex, $n(\%)$ & $46(64)$ & $44(64)$ \\
\hline Secondary level education, $n(\%)$ & $45(63)$ & $45(66)$ \\
\hline Degree level education, $n(\%)$ & $26(36)$ & $20(29)$ \\
\hline Mean (SD) weight, kg & $91.6(13.3)$ & $94.4(14.2)$ \\
\hline Mean (SD) waist circumference, cm & $104.2(11.3)$ & $106.4(10.5)$ \\
\hline Meeting physical activity target, $n(\%)$ & $24(33.3)$ & $20(29.0)$ \\
\hline
\end{tabular}


Table 2. Outcomes at 6 months.

\begin{tabular}{lccc} 
& $\begin{array}{c}\text { Intervention } \\
(n=72)\end{array}$ & $\begin{array}{c}\text { Control } \\
(n=69)\end{array}$ & $\begin{array}{c}\text { Size of effect/ } \\
\text { significance }\end{array}$ \\
\hline $\begin{array}{l}\text { Total (\%) achieving } \\
5 \% \text { weight loss }\end{array}$ & 17 & 5 & $\mathrm{OR}=4.0 ;$ \\
& $(23.6)$ & $(7.2)$ & $95 \% \mathrm{Cl}=1.4$ to 11.4 \\
\hline $\begin{array}{l}\text { Total (\%) achieving } \\
150 \text { minutes activity }\end{array}$ & 27 & 19 & $\mathrm{OR}=1.6$ \\
\hline $\begin{array}{l}\text { Number (\%) achieving } \\
150 \text { minutes moderate activity, } \\
\text { but not achieving this at baseline }\end{array}$ & $(37.5)$ & $(27.5)$ & $95 \% \mathrm{Cl}=0.7$ to 3.8 \\
\hline Mean (SD) weight, $\mathrm{kg}^{\mathrm{b}}$ & 10 & 5 & $\mathrm{OR}=2.1$ \\
& 91.3 & $(7.2)$ & $95 \% \mathrm{Cl}=0.7$ to 6.4 \\
\hline $\begin{array}{l}\text { Mean (SD) waist } \\
\text { circumference, } \mathrm{cm}^{\mathrm{b}}\end{array}$ & $(13.7)$ & $(15.0)$ & $95 \% \mathrm{Cl}=0.2$ to 2.4 \\
\hline
\end{tabular}

$O R=$ odds ratio. ${ }^{a}$ Analysis takes into account baseline value as an integral part of the measure. ${ }^{b}$ Analysis and means (but not proportions) adjusted for value of baseline dependent variable.

provides detailed costings. No important adverse events were reported in either group.

\section{DISCUSSION}

\section{Summary of main findings}

The study shows that personnel recruited from outside the existing health service, with brief training in motivational interviewing, can help people to achieve a $5 \%$ weight-loss target. The results suggest an additional small increase in the proportion achieving a clinically meaningful change in physical activity, although this was not significant.

\section{Strengths and limitations of the study}

The study's main strengths were in its pragmatic nature and setting and broad inclusion criteria, which increase the likely generalisability of the results. ${ }^{21}$ The effect was estimated in a practice setting, with realistic dropout and non-adherence to treatment taken into account using intention-to-treat analysis. A high retention rate was achieved in both groups, with $88 \%$ of men and $76 \%$ of women completing the intervention. The relatively high degree of engagement and retention of men is worth noting. The collection of objective measures (weight and waist) was blinded to group allocation. However, costs were not rigorously estimated or analysed. The study had insufficient power to detect the (smaller than expected) changes in proportions achieving the physical activity target. This was compounded by the fact that the response rate for physical activity questionnaires was low. In addition, the self-report of physical activity has low sensitivity and limited validity (a tendency for over-estimation and low-to-moderate correlations with objective measures). ${ }^{22}$ Generalisability may also be limited by the self-selecting nature of the sample (around 57\% agreed to take part), although it should be noted that this kind of self-selection would also apply to a GPmediated offer of referral to a weight-loss intervention.
The practices involved were semi-rural with a broad population demographic, but with only limited representation from ethnic minority groups. A larger study would therefore be needed to establish generalisability to other UK practices.

A further limitation of this study is that it is not possible to say whether the effects found would persist beyond 6 months. It is known that the effects of moreintensive interventions, such as the Finnish Diabetes Prevention Study, can be largely maintained for as much as 7 years. ${ }^{4}$ However, less-intensive behavioural interventions are less effective and often lose their effects over time, ${ }^{23-25}$ and weight-loss interventions often suffer from high dropout. It could be hypothesised that the type of intervention used in the present study should provide good maintenance of weight loss, as motivational interviewing aims to equip people with the cognitive tools to make and sustain behaviour change. ${ }^{8}$ This is supported by review evidence that effect sizes in nine trials of motivational interviewing, involving 1500 people, did not significantly decrease between 20 and 67 weeks. ${ }^{15}$ However, the existence of longer-term, generalisable benefits for this specific application of motivational interviewing needs to be established in larger clinical trials.

\section{Comparison with existing literature}

This study's findings are consistent with other trials of motivational interviewing, which have produced significant weight loss and physical activity increases. ${ }^{15,16,26}$

The Finnish Diabetes Prevention Study suggests that weight is the dominant behavioural predictor of progression to diabetes. ${ }^{3}$ In their study, people with hyperglycaemia achieving an initial $5 \%$ weight loss were $55 \%$ less likely (hazard ratio 0.45 ) to develop type 2 diabetes within 7 years. ${ }^{3}$ In the present study, the difference in the proportions achieving $5 \%$ weight loss $(17 \%)$ was less than that achieved in the Finnish trial (30\%). One reason for this could be that the participants did not have pre-diabetes, which may increase motivation for behaviour change. ${ }^{27}$ However, it is likely that the reduced intensity of intervention was also a factor. ${ }^{15,16}$

This study therefore suggests that in devising lowercost, simpler interventions, there may be a trade off between effect size and cost. The costs per patient of the study (estimated £263) were well below those of the US and Finnish diabetes-prevention programmes (£1796 ${ }^{28}$ and $£ 2116^{29}$ respectively, when inflated to 2007 prices). A comparison with pharmaceutical approaches is also possible. The NNT for 2 years to achieve $5 \%$ weight loss using orlistat is $6(95 \% \mathrm{Cl}=4$ to 14), with an estimated treatment cost (at 2007 prices) of $£ 1759$ per patient. ${ }^{30}$ However, it is worth 
noting that the present study did not set out to formally measure or compare cost-effectiveness, and these figures are presented mainly to contextualise this study as a lower-cost approach.

\section{Implications for future research and clinical practice}

Future research in this area should use lessburdensome and more objective measures of physical activity (for example, a simpler questionnaire and accelerometers). The longer-term effectiveness and cost-effectiveness of a range of well-defined intervention approaches, with different levels of intensity, needs to be established to inform policy decisions and future clinical practice in this area. Monitoring of intervention processes (both intervention delivery and intra-individual/ psychological processes) and the relation of processes to outcomes is also essential for advancing the science and methods of behavioural intervention. ${ }^{31}$

The current study has shown that it is feasible to deliver a low-cost intervention, which produces meaningful changes in weight, at a level which, if sustained, can modify progression to diabetes. The intervention can be delivered in primary care, without creating an excessive drain on NHS monetary or personnel resources.

\section{Funding body}

The study was funded by Mid Devon Primary Care Trust. Two of the authors (Lucy O' Loughlin, Sandra Holland) were employed by this trust at the time of the study. Their involvement in the study does not however cause any conflict of interest. The time input from other authors was largely subsidised by their employing organisations. Colin Greaves and Anna Steele are also funded by NHS National Institute for Health Research fellowship grants

\section{Ethical approval}

North and East Devon Local Research Ethics Committee (05/Q2102/40)

\section{Competing interests}

The authors have stated that there are none

\section{Acknowledgements}

The authors acknowledge the advice of Dr Rod Taylor of the Peninsula Medical School Clinical Trials Methodology Unit on statistical matters, and would like to thank all the participants and the counsellors and health professionals who contributed their time and effort to this study.

\section{Discuss this article}

Contribute and read comments about this article on the Discussion Forum: http://www.rcgp.org.uk/bjgp-discuss

\section{REFERENCES}

1. Knowler WC, Barrett-Connor E, et al. Diabetes Prevention Program Research Group. Reduction in the incidence of type 2 diabetes with lifestyle intervention or metformin. N Eng J Med 2002; 346(6): 393-403.

2. Pan XR, Li GW, Hu YH, et al. Effects of diet and exercise in preventing NIDDM in people with impaired glucose tolerance. The Da Qing IGT and Diabetes Study. Diabetes Care 1997; 20(4): 537-544.

3. Tuomilehto J, Lindstrom J, Eriksson JG, et al. Prevention of type 2 diabetes mellitus by changes in lifestyle among subjects with impaired glucose tolerance. N Eng J Med 2001; 344(18): 1343-1350.

4. Lindstrom J, Ilanne-Parikka P, Peltonen M, et al. Sustained reduction in the incidence of type 2 diabetes by lifestyle intervention: follow-up of the
Finnish Diabetes Prevention Study. Lancet 2006; 368(9548): 1673-1679.

5. Gabir M, Hanson RL, Dabelea D, et al. The 1997 American Diabetes Association and 1999 World Health Organization criteria for hyperglycemia in the diagnosis and prediction of diabetes. Diabetes Care 2000; 23(8): 1108-1112.

6. Heneghan C, Thompson M, Perera R. Prevention of diabetes. BMJ 2006; 333(7572): 764-765.

7. Goldberg RB. Lifestyle interventions to prevent type 2 diabetes. Lancet 2006; 368(9548): 1634-1936.

8. Miller WR, Rollnick S. Motivational interviewing: preparing people for change. 2nd edn. New York, NY: Guildford Press, 2002.

9. Knight KM, McGowan L, Dickens C, Bundy C. A systematic review of motivational interviewing in physical health care settings. $\mathrm{Br} J$ Health Psychol 2006; 11 (Pt 2): 319-332.

10. Miller WR. Motivational interviewing in service to health promotion. Am J Health Promot 2004; 18(3): A1-A10.

11. Vanwormer JJ, Boucher JL. Motivational interviewing and diet modification: a review of the evidence. Diabetes Educ 2004; 30(3): 404-419.

12. Motivational interviewing: MINT trainers. Motivational Interviewing Network of Trainers. http://motivationalinterview. org/training/trainers.html (accessed 29 Apr 2008).

13. Bundy C. Changing behaviour: using motivational interviewing techniques. J R Soc Med 2004; 97 Suppl 44: 43-47.

14. Lane $\mathrm{C}$, Huws-Thomas M, Hood $\mathrm{K}$, et al. Measuring adaptations of motivational interviewing: the development and validation of the Behavior Change Counseling Index (BECCI). Patient Educ Couns 2005; 56(2): 166-173.

15. Burke BL, Arkowitz H, Menchola M. The efficacy of motivational interviewing: a meta-analysis of controlled clinical trials. J Consult Clin Psychol 2003; 71(5): 843-861.

16. Rubak S, Sandboek A, Lauritzen T, Christensen B. Motivational interviewing: a systematic review and meta-analysis. Br J Gen Pract 2005; 55(513): 305-312.

17. American Diabetes Association. Screening for diabetes. Diabetes Care 2004; 27 (Suppl 1): S11-S14.

18. Department of Health. Choosing health: making healthier choices easier. London: Department of Health, 2004.

19. Wang J. Waist circumference: a simple, inexpensive, and reliable tool that should be included as part of physical examinations in the doctor's office. Am J Clin Nutr 2003; 78(5): 902-903.

20. Kriska AM, Caspersen CJ. Introduction to the collection of physical activity questionnaires. Med Sci Sports Exerc 1997; 29: S73-S78.

21. Roland $M$, Torgerson DJ. Understanding controlled trials: what are pragmatic trials? BMJ 1998; 316(7165): 285.

22. Shephard RJ. Limits to the measurement of habitual physical activity by questionnaires. Br J Sports Med 2003; 37(3): 197-206.

23. Renders CM, Renders CM, Valk GD, et al. Interventions to improve the management of diabetes mellitus in primary care, outpatient and community settings. Cochrane Database Syst Rev 2001; CD001481.

24. Avenell A, Broom J, Brown TJ, et al. Systematic review of the long-term effects and economic consequences of treatments for obesity and implications for health improvement. Health Technol Assess 2004; 8(21): iii-iv, 1-182.

25. Pryke R, Docherty A. Obesity in primary care: evidence for advising weight constancy rather than weight loss in unsuccessful dieters. Br J Gen Pract 2008; 58(547): 112-117.

26. Hardcastle S, Taylor A, Bailey M, Castle R. A randomised controlled trial on the effectiveness of a primary health care based counselling intervention on physical activity, diet and CHD risk factors. Patient Educ Couns 2008; 70(1): 31-39.

27. Evans PH, Greaves CJ, Winder R, et al. Development of an educational 'toolkit' for health professionals and their patients with prediabetes: the WAKEUP study (Ways of Addressing Knowledge Education and Understanding in Pre-diabetes). Diabet Med 2006; 24(7): 770-777.

28. Lindgren $P$, Lindström J, Tuomilehto J, et al. Lifestyle intervention to prevent diabetes in men and women with impaired glucose tolerance is cost-effective. Int J Technol Assess Health Care 2007; 23(2): 177-183.

29. The Diabetes Prevention Programme Research Group. Within-trial costeffectiveness of lifestyle intervention or metformin for the primary prevention of type 2 diabetes. Diabetes Care 2003; 26(9): 2518-2523.

30. Foxcroft DR, Milne R. Orlistat for the treatment of obesity: rapid review and cost-effectiveness model. Obes Rev 2000; 1(2): 121-126.

31. Michie S, Abraham C. Interventions to change health behaviours: evidence-based or evidence-inspired. Psychol Health 2004; 19(1): 29-50. 


\section{Appendix 1. Estimated intervention costs.}

Cost item

Cost $(£)$

Total project worker time (inclusive of on-costs, supervision, and training time) 1015 hours @ £10.57

10728.55

Management team/supervision time: 52 weeks at 2 hours per week @ £19.98/h (including on-costs)

2077.92

Room cost/reception cover (including hospital overheads at 100\%)

Stationery

135.00

Telephone (estimated)

500.00

Secretarial support: 52 hours (1/week) @ £9.80 (including on-costs)

509.60

Training (motivational interviewing), as charged

1500.00

Training (internal) 4 hours $\times 4$ trainers $\times$ (average) $£ 34.80 / h$ (including on-costs) 556.80

GP costs (including on-costs) for identification and referral of patients (5 minutes administrator $£ 14.60 /$ hour 451.38 and 5 minutes GP time $£ 60.63 /$ hour $) \times 72$ patients

Total cost

18940.97

Cost per intervention 